\title{
Sensing of RNA stress by mTORC1 drives autoinflammation
}

\author{
Min Ae Lee-Kirsch
}

Department of Pediatrics, Medizinische Fakultät Carl Gustav Carus, Technische Universität Dresden, Dresden, Germany.

\begin{abstract}
Loss-of-function mutations in SKIV2L underlie trichohepatoenteric syndrome (THES2), a rare inborn error of immunity characterized by diarrhea, skin lesions, brittle hair, and immunodeficiency. SKIV2L is part of a multiprotein complex required for exosome-mediated RNA surveillance through RNA decay. In this issue of the $J C l$, Yang et al. delineate a mechanism underlying autoinflammatory skin disease in Skiv2l-deficient mice. Thus, a lack of SKIV2L activates mTORC1 signaling in keratinocytes and $\mathrm{T}$ cells, impeding skin barrier integrity and $\mathrm{T}$ cell homeostasis. Interestingly, treatment with the mTOR inhibitor rapamycin improves skin symptoms in Skiv2l-deficient mice, suggesting a possible therapeutic avenue for patients with THES2.
\end{abstract}

\section{RNA metabolism and RNA sensing}

The human genome is transcribed to generate an extraordinary diversity of RNA, which is subject to complex regulation to maintain cellular homeostasis. The turnover and quality control of ribosome-associated mRNA are controlled by the cytoplasmic RNA exosome, an RNA degradation machinery acting in concert with the super-killer (SKI) complex (1). The SKI complex consists of the SKIV2L helicase, two subunits of the WD-repeat protein WDR61, and the tetratricopeptide repeat motif containing protein TTC37 (2). It associates with the $80 \mathrm{~S}$ ribosome and extracts mRNA that is no longer needed or recognized as being faulty to initiate ribonucleolytic degradation from the 3 ' end by the RNA exosome (Figure 1) $(3,4)$.

RNA metabolism is also critical for immune homeostasis, as self-RNA occurring in the wrong place at the wrong time can turn into a danger signal that triggers immune responses leading to autoin- flammation or autoimmunity (5). Sensing of viral RNA by receptors of the innate immune system is an essential strategy in antiviral immunity (6). A central challenge for the host cell is to discriminate between harmful foreign RNA and selfRNA. Ligand specificity of RNA sensors, such as the cytosolic RNA helicases RIG-I and MDA5, relies largely on unique structural properties of viral RNA $(5,6)$. Thus, while RIG-I senses 5 '-triphosphate-RNA or 5'-diphosphate-RNA, MDA5 recognizes long, double-stranded RNA (7-9). Engagement of these RNA sensors triggers type I interferon (IFN) signaling, resulting in the activation of numerous antiviral transcriptional programs $(5,6)$. The processes of RNA metabolism and RNA sensing must be tightly regulated to avoid accumulation of potentially immunostimulatory self-RNA and to prevent inappropriate innate immune activation. The SKIV2L RNA exosome has been shown to degrade immunostimulatory self-RNA arising as a cleavage product of the endonuclease

\section{Related Article: https://doi.org/10.1172/JCl146176}

Conflict of interest: The author has declared that no conflict of interest exists.

Copyright: ( 2022 , Lee-Kirsch et al. This is an open access article published under the terms of the Creative Commons Attribution 4.0 International License.

Reference information: / Clin Invest. 2022;132(2):e156119. https://doi.org/10.1172/JCI156119.

IRE-1 during endoplasmic reticulum stress (10), thereby limiting type I IFN-dependent immune activation. However, the role of the SKIV2L RNA exosome for immune homeostasis in the absence of ER stress remains poorly understood.

\section{RNA stress due to SKIV2L deficiency activates mTORC1 signaling}

Loss-of-function mutations in SKIV2L cause trichohepatoenteric syndrome (THES2), a rare inborn error of immunity characterized by intrauterine growth retardation, early-onset chronic diarrhea, brittle hair with trichorrhexis nodosa, skin lesions, and immunodeficiency $(11,12)$. In this issue of the JCI, Yang et al. (13) set out to dissect the functional consequences of Skiv2l deficiency in mice. To bypass embryonic lethality of mice with complete Skiv $2 l$ knockout, they turned to mice with tamoxifen-inducible whole-body deletion of Skiv2l. These mice developed inflammatory skin lesions due to impaired epidermal stratification with loss of epidermal barrier integrity. Skin-specific deletion of Skiv2l also led to epidermal hyperplasia with defective hair morphogenesis, recapitulating the human disease phenotype. Interestingly, these phenotypic changes were not accompanied by activation of the type I IFN axis, as shown by a lack of expression of IFN-stimulated genes in Skiv2l-deficient epidermis or primary keratinocytes. Moreover, mice with myeloid-specific Skiv2l knockout did not show any skin pathology or signs of inflammation, suggesting that skin pathology in Skiv2l deficiency occurs independent of the hematopoietic system. Together, these findings indicate a cell-intrinsic mechanism by which the SKI-associated RNA exosome regulates keratinocyte function and which is required for skin barrier integrity independent of type I IFN signaling.

Transcriptional profiling of epidermal tissue of mice with keratinocytespecific Skiv2l deletion revealed enrich- 
A Normal cell physiology

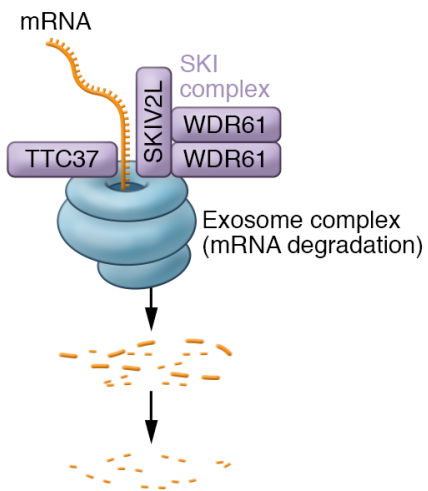

Ribonucleotides
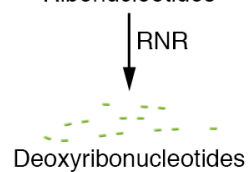

Deoxyribonucleotides 'Homeostatic control?

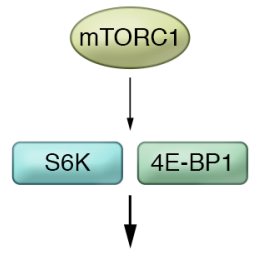

Anabolic processes

Cell growth
B SKIV2L deficiency
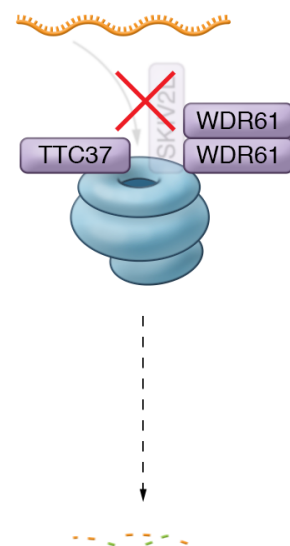

Imbalanced nucleotide metabolism?

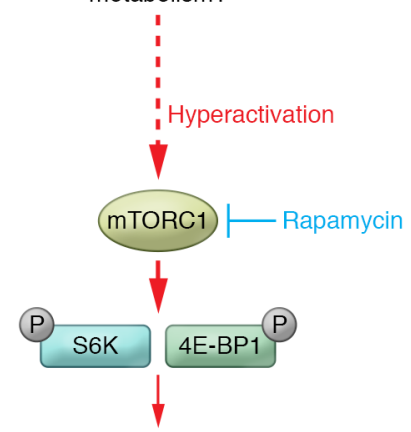

Uncontrolled proliferation Inflammatory responses
Figure 1. A model for mTORC1 signaling in SKIV2L deficiency that leads to uncontrolled proliferation and immune activation. (A) The turnover and quality control of ribosome-associated mRNA are controlled by the cytoplasmic RNA exosome, which acts as a molecular shredder by degrading mRNA from the $3^{\prime}$ end. The RNA exosome is assisted by the SKI complex. The SKIV2L helicase unwinds RNA and, together with the TPR motif-containing protein TTC37 and two subunits of the WD-repeat protein WDR61, initiates ribonucleolytic degradation. The mTORC1 complex senses nutrient deprivation, which induces activation of downstream targets S6K and 4E-BP1 by phosphorylation. mTORC1 signaling promotes a broad range of anabolic processes, including cell growth and proliferation, which require a sufficient supply of deoxyribonucleotides for DNA synthesis. (B) Aberrant mTORC1 signaling in SKIV2L deficiency may be triggered by sensing changes in cellular concentrations of deoxyribonucleotides, which are produced from ribonucleotides by the action of ribonucleotide reductase (RNR). Unabated mTORC1 activity in keratinocytes and T cells leads to hyperproliferation with secondary inflammatory responses. Rapamycin inhibits the protein kinase mTOR, core component of the mTORC1 complex, and may therefore be of therapeutic benefit in patients with SKIV2L deficiency. ment of genes acting in the mTORC1 signaling pathway. The mTORC1 complex, a multiprotein assembly consisting of the serine/threonine protein kinase mTOR, raptor, mLST8, PRAS40, and DEPTOR, acts as a central hub that integrates nutrient signaling pathways to promote cell growth (14). The authors confirmed activation of mTORC1 signaling in Skiv2ldeficient keratinocytes by demonstrating enhanced phosphorylation of ribosomal S6 kinase (S6K) and 4E-BP1, the key downstream effectors of the mTORC1 pathway (14), along with increased global protein synthesis. Skiv2l-deficient skin lesions showed $\mathrm{T}$ cell infiltrates and these $\mathrm{T}$ cells were chronically activated as shown by increased proliferation in response to ex vivo stimulation with antiCD3/CD28. Similar to keratinocytes, $\mathrm{T}$ cell hyperactivation was accompanied by increased phosphorylation of $\mathrm{S} 6 \mathrm{~K}$ and 4E-BP1, consistent with an mTORC1dependent, cell-intrinsic role for Skiv2l in $\mathrm{T}$ cell homeostasis. Interestingly, increased epidermal phospho-S6K staining was also observed in lesional skin of a patient with THES2 who presented with failure to thrive, diarrhea, trichorrhexis nodosa, erythematous rash, elevated liver enzymes, and glomerulonephritis, indicating activation of the mTORC1 pathway also in human SKIV2L deficiency. Finally, the authors demonstrated that both systemic and topical treatment of Skiv2l-deficient mice with the mTOR inhibitor rapamycin ameliorated epidermal hyperplasia and skin inflammation.

\section{Conclusions and implications}

The mTORC1 pathway promotes cell growth through activation of anabolic processes, including the biosynthesis of proteins, lipids, and nucleotides, as well as through cell-cycle acceleration (14). Thus, enhanced mTORC1 signaling observed in keratinocytes and $\mathrm{T}$ cells of Skiv2l-deficient mice provides a mechanistic explanation for uncontrolled hyperproliferation, which is initiated cell-autonomously, resulting in the loss of skin barrier integrity and $\mathrm{T}$ cell homeostasis (Figure 1 ). The T cell phenotype described in Skiv2l-deficient mice is intriguing, because some patients with THES2 develop reactive hemophagocytic syndrome, a hyperinflammatory state caused by hyperactivation of $\mathrm{T}$ cells and macrophages $(12,15)$. Moreover, the findings by Yang et al. indicate that a lack of cytoplasmic RNA quality control due to dysfunction of the SKI-associated RNA exosome is sensed by mTORC1, although the exact nature of the metabolites that are actually sensed by mTORC1 under these circumstances is still unknown.

Cell growth and proliferation requires increased DNA replication, which depends on a sufficient supply of nucleotides (deoxyribonucleotides), the building blocks of DNA synthesis (14). An increased demand for nucleotides is also controlled downstream of mTORC1 through stimulation of de novo nucleotide biosynthesis (16-19). However, the major pathway for biosynthesis of DNA precursors is mediated by ribonucleotide reductase, which generates deoxyribonucleotides from ribonucleotides (20), the end product of the RNA exosome. Thus, while RNA degradation is an inherent step in RNA quality control mechanisms, it also contributes to 
the recycling of the nucleotide pool in the cell (Figure 1). However, whether changes in cellular nucleotide concentrations underlie mTORC1 signaling in SKIV2L deficiency remains to be investigated.

Interestingly, the authors describe a lack of type I IFN activation in skin and blood of Skiv2l-deficient mice, arguing against a pathogenetic role of RIG-Idependent innate immune activation by unprocessed self-RNA in SKIV2L deficiency. This result contrasts with work by Eckard et al., which demonstrates an IFN signature in the blood of two patients with THES2, but not in three patients with THES1 who carried mutations in the SKI complex component TTC37 (10, 21), despite having indistinguishable clinical features. The findings by Yang et al. (13) are also in line with a clinical report, demonstrating absence of an IFN signature in a patient with THES2 (15). Nonetheless, a moderately increased expression of MX1, an IFN-regulated gene, was found in skin lesions of the patient studied by Yang et al. (13). The type I IFN activation observed in patients with THES 2 might act as a permissive factor rather than as the primary cause of inflammation. This notion is also supported by the therapeutic efficacy of rapamycin in Skiv2l-deficient mice. Patients with THES develop intractable diarrhea commonly leading to failure to thrive (11, 12). Although mice with Skiv2l deficiency do not exhibit intestinal symptoms, it is possible that a loss of intestinal barrier integrity due to aberrant mTORC1 signaling may account for intestinal dysfunction in patients with THES2. As such, rapamycin may provide a promising and potentially curative therapy for these patients.
Perturbations of the mTORC1 pathway have been implicated in a variety of human diseases, including common autoimmune diseases such as systemic lupus erythematosus (22). Given the genetic and phenotypic heterogeneity of these complex diseases, mTORC1 hyperactivation may represent a useful endotype, enabling further stratification of patients based on mechanistic insight.

\section{Acknowledgments}

MLK is supported by grants from the Deutsche Forschungsgemeinschaft (KFO249 160548243 and CRC237 369799452/B21).

Address correspondence to: $\mathrm{Min} \mathrm{Ae}$ Lee-Kirsch, Department of Pediatrics, Medizinische Fakultät Carl Gustav Carus, Technische Universität Dresden, Fetscherstr. 74, 01307 Dresden, Germany. Phone: 49.351.4586887; Email: minae. lee-kirsch@uniklinikum-dresden.de.

1. Houseley J, et al. RNA-quality control by the exosome. Nat Rev Mol Cell Biol. 2006;7(7):529-539.

2. Halbach F, et al. The yeast ski complex: crystal structure and RNA channeling to the exosome complex. Cell. 2013;154(4):814-826.

3. Schmidt C, et al. The cryo-EM structure of a ribosome-Ski2-Ski3-Ski8 helicase complex. Science. 2016;354(6318):1431-1433.

4. Zinoviev A, et al. Extraction of mRNA from stalled ribosomes by the ski complex. Mol Cell. 2020;77(6):1340-1349.

5. Schlee M, Hartmann G. Discriminating self from non-self in nucleic acid sensing. Nat Rev Immunol. 2016;16(9):566-580.

6. Rehwinkel J, Gack MU. RIG-I-like receptors: their regulation and roles in RNA sensing. Nat Rev Immunol. 2020;20(9):537-551.

7. Hornung V, et al. 5'-Triphosphate RNA is the ligand for RIG-I. Science. 2006;314(5801):994-997.

8. Goubau D, et al. Antiviral immunity via RIG-I- mediated recognition of RNA bearing 5'-diphosphates. Nature. 2014;514(7522):372-375.

9. Kato H, et al. Differential roles of MDA5 and RIG-I helicases in the recognition of RNA viruses. Nature. 2006;441(7089):101-105.

10. Eckard SC, et al. The SKIV2L RNA exosome limits activation of the RIG-I-like receptors. Nat Immunol. 2014;15(9):839-845.

11. Fabre A, et al. SKIV2L mutations cause syndromic diarrhea, or trichohepatoenteric syndrome. Am J Hum Genet. 2012;90(4):689-692.

12. Vély F, et al. Combined immunodeficiency in patients with trichohepatoenteric syndrome. Front Immunol. 2018;9:1036.

13. Yang K, et al. Cytoplasmic RNA quality control failure engages mTORC1-mediated autoinflammatory disease. JClin Invest. 2022;132(2):e146176.

14. Liu GY, Sabatini DM. mTOR at the nexus of nutrition, growth, ageing and disease. Nat Rev Mol Cell Biol. 2020;21(4):183-203.

15. Hiejima E, et al. Tricho-hepato-enteric syndrome with novel SKIV2L gene mutations: a case report. Medicine (Baltimore). 2017;96(46):e8601.

16. Robitaille AM, et al. Quantitative phosphoproteomics reveal mTORC1 activates de novo pyrimidine synthesis. Science. 2013;339(6125):1320-1323.

17. Ben-Sahra I, et al. Stimulation of de novo pyrimidine synthesis by growth signaling through mTOR and S6K1. Science. 2013;339(6125):1323-1328.

18. Ben-Sahra I, et al. mTORC1 induces purine synthesis through control of the mitochondrial tetrahydrofolate cycle. Science. 2016;351(6274):728-733.

19. Düvel K, et al. Activation of a metabolic gene regulatory network downstream of mTOR complex 1. Mol Cell. 2010;39(2):171-183.

20. Reichard P. Ribonucleotide reductase and deoxyribonucleotide pools. Basic Life Sci. 1985;31:33-45.

21. Hartley JL, et al. Mutations in TTC37 cause trichohepatoenteric syndrome (phenotypic diarrhea of infancy). Gastroenterology. 2010;138(7):2388-2398.

22. Sharabi A, Tsokos GC. T cell metabolism: new insights in systemic lupus erythematosus pathogenesis and therapy. Nat Rev Rheumatol. 2020;16(2):100-112. 